\title{
Propiedades físicas de naranja agria cocristalizada: efecto del pH, sólidos solubles y zumo adicionado ${ }^{1}$
}

\author{
Physical properties of co-crystallized bitter orange: effect of $\mathrm{pH}$, soluble solids \\ and added juice
}

\author{
Ricardo Andrade-Pizarro ${ }^{2}$, Kevin Blanquicett-González ${ }^{3}$, Rafael Rangel-Terraza ${ }^{3}$
}

\begin{abstract}
Resumen
El objetivo del presente trabajo fue evaluar las condiciones de cocristalización del zumo de naranja agria con sacarosa, sobre las propiedades fisicoquímicas del producto obtenido. El zumo de naranja agria fue obtenido con un exprimidor mecánico y concentrado en un rota-evaporador. Se preparó un jarabe de sacarosa a $70{ }^{\circ} \mathrm{Brix}$, el cual fue sometido a calentamiento y agitación de $1000 \mathrm{rpm}$, hasta que se observó una coloración blanca $\left(118{ }^{\circ} \mathrm{C}\right)$. El zumo de naranja agria se adicionó al jarabe, y la mezcla fue sometida a agitación constante de 600 rpm, hasta observar la formación de un material sólido particulado. Los cocristales fueron secados, molturados y tamizados. A los cocristales secos se les determinó: humedad, densidad aparente, solubilidad, actividad de agua y el ángulo de reposo. Una alta proporción de zumo de naranja agria adicionada (20\%) y bajo contenido de sólidos solubles (50 ${ }^{\circ}$ Brix) producen cocristales con bajo contenido de humedad $(2,59 \%)$, actividad de agua $(0,52)$ y tiempo de solubilidad $(69,4 \mathrm{~s})$. El zumo de naranja agria concentrado con un $\mathrm{pH}$ de 4,5, produjo cocristales con bajos contenidos de humedad $(1,96 \%)$. Los cocristales de zumo de naranja agria mostraron buenas características de reconstitución (alta solubilidad); sin embargo, presentaron alta humedad $(2,5$ a $4,5 \%)$ y actividad de agua $(0,508$ a 0,798$)$.
\end{abstract}

Palabras clave: Citrus aurantium L., encapsulación, cocristalización.

\begin{abstract}
The aim of this study was to evaluate the conditions of co-crystallization of bitter orange juice with sucrose on the physicochemical properties of the product. Bitter orange juice was obtained with a mechanical juicer and concentrated on a rotary evaporator. Sucrose syrup $70^{\circ}$ Brix was subjected to heating and stirring of $1000 \mathrm{rpm}$, until a white color $\left(118^{\circ} \mathrm{C}\right)$ was observed. Bitter orange juice was added to the syrup, and the mixture was subjected to constant stirring of $600 \mathrm{rpm}$, to observe the formation of a particulate solid material. The co-crystals were dried, grinded and sieved. A dry co-crystal was determined: moisture, bulk density, solubility, water activity and repose angle. High proportion of bitter orange juice added $(20 \%)$ and low content of soluble solids $\left(50{ }^{\circ} \mathrm{Brix}\right)$ produced co-crystals with low moisture content $(2.59 \%)$, water activity (0.52) and solubility time (69.4 s). High $\mathrm{pH}(4.5)$ of bitter orange juice concentrate
\end{abstract}

1 Recibido: 3 de mayo, 2016. Aceptado:10 de octubre, 2016. Este trabajo formó parte de la tesis de grado de Ingeniería de Alimentos de Kevin Blanquicett González y Rafael Rangel Terraza, de la Universidad de Córdoba, Montería, Colombia.

2 Universidad de Córdoba, Facultad de Ingeniería, Grupo de Investigación de Propiedades y Procesamiento de Alimentos (GIPPAL). Km 12 Vía Cereté-Ciénaga de Oro, Colombia.rdandrade@correo.unicordoba.edu.co

3 Universidad de Córdoba, Programa de Ingeniería de Alimentos, Km 12 Vía Cereté-Ciénaga de Oro, Colombia. kevincamilobg@yahoo.com, rafarangel14@hotmail.com 
produced co-crystals with low moisture contents (1.96\%). The co-crystals of bitter orange juice showed good reconstitution characteristics (high solubility); however, these had high humidity (2.5 to $4.5 \%$ ) and water activity (0.508 to 0.798$)$.

Keywords: Citrus aurantium L., encapsulation, co-crystallization.

\section{Introducción}

La encapsulación en la industria alimentaria es un método frecuentemente utilizado para mejorar las propiedades de manejo y producción de algunos alimentos, conservando su valor nutricional y sensorial (vitaminas, minerales, aromas, sabores, etc). Existe una amplia variedad de técnicas para realizar este proceso, dentro de los cuales se encuentra la cocristalización. En la cocristalización se modifica la estructura sólida, monocíclica y densa de los cristales puros de sacarosa, para obtener un aglomerado irregular y poroso que facilite la incorporación de un ingrediente activo (Chen et al., 1988; Bhandari et al., 1998; Sardar y Singhal, 2013; Sardar et al., 2013). Esta técnica mejora la solubilidad, humectabilidad, homogeneidad, dispersabilidad, hidratación, la propiedad antiapelmazante y la capacidad de flujo del ingrediente activo encapsulado (Beristain et al., 1996; Bhandari y Hartel, 2002). Debido a su estructura, los productos cocristalizados con azúcar ofrecen características que los hacen atractivos para las industrias de dulces y farmacéutica (Awad y Chen, 1993; López-Córdoba et al., 2014).

Diversos componentes pueden ser encapsulados, entre los cuales se encuentran: ingredientes activos, sustancias volátiles, emulsionantes, ácidos, entre otros (Chen et al., 1988; López-Córdoba et al., 2015). Actualmente, los zumos de frutas son fuentes importantes de ingredientes activos, dentro de los cuales el zumo de naranja agria se destaca por su sabor único, beneficios en la salud y contenido de vitamina C (Alonso, 2004). Diversos estudios han establecido que en la cocristalización de zumos de frutas es importante evaluar diferentes parámetros, entre los que se encuentran: pH (Beristain et al., 1994; Astolfi et al., 2005), la relación de zumo de fruta-jarabe de sacarosa (Beristain et al., 1994; Bhandari et al., 1998) y la concentración del zumo de fruta (Astolfi et al., 2005; Montes et al., 2006).

Encapsular con naranja agria es una de las tendencias actuales en la industria alimentaria, principalmente en la elaboración de productos de origen natural y con alto valor nutricional (Astray et al., 2009). Por lo anterior, se planteó como objetivo del presente trabajo, evaluar las condiciones de cocristalización del zumo de naranja agria con sacarosa, sobre de las propiedades fisicoquímicas del producto obtenido.

\section{Materiales y métodos}

\section{Materia prima}

Los frutos de naranja agria (Citrus aurantium L.) se recolectaron en la finca San Antonio perteneciente al corregimiento de Malagana de Ciénaga de Oro, Monteria, Colombia, con un estado de maduración de 2 de acuerdo con Barón y Villa (2013). Las naranjas se enjuagaron con agua a presión y se desinfectaron por inmersión en cloro diluido al $0,5 \%$ durante $10 \mathrm{~min}$. 


\section{Obtención del zumo de naranja agria}

La obtención del zumo de naranja agria se realizó con un exprimidor mecánico, filtrándolo con tela fina. El zumo de naranja presentó una humedad del 90,6\%, sólidos solubles de 9,7 ${ }^{\circ}$ Brix, ácido cítrico de 4,13\%, pH de 2,94 y vitamina C de 49,1 mg/100g.

\section{Cocristalización del zumo}

Al zumo de naranja agria se le ajustó el pH hasta 3,8; 4,15 y 4,5, empleando hidróxido de calcio. Luego se llevó a un rota-evaporador hasta la concentración de sólidos solubles de 50, 55 y 60 Brix. El zumo concentrado se etiquetó y almacenó en refrigeración.

Para preparar los jarabes de sacarosa se pesaron $300 \mathrm{~g}$ de azúcar y $50 \mathrm{ml}$ de agua, con el fin de obtener una solución concentrada de $70{ }^{\circ}$ Brix. Esta se sometió a calentamiento en una plancha eléctrica con agitación magnética a $1000 \mathrm{rpm}$, hasta observar una coloración blanca ( 30 min), aproximadamente a una temperatura de 118 ${ }^{\circ} \mathrm{C}$, la cual indicó el grado de sobresaturación requerido para que ocurriera la cristalización espontánea al enfriarse. En este punto el jarabe se retiró de la fuente de calentamiento y se le añadió los porcentajes de zumo concentrado establecidos. La mezcla se sometió a agitación constante de $600 \mathrm{rpm}$, usando un agitador mecánico portátil dotado con aspas metálicas rectas de 4,5 cm de diámetro. Al observar la formación de un material sólido particulado, la agitación se detuvo.

El material particulado se separó y sometió a un proceso de secado a $50{ }^{\circ} \mathrm{C}$ en una estufa al vacío durante 24 $\mathrm{h}$, seguido por una molturación y cribado utilizando un colador comercial con un diámetro de $1 \mathrm{~mm}$. Finalmente, el producto se depositó en bolsas de polietileno transparente (Calibre 200) y se almacenaron hasta su posterior análisis.

\section{Propiedades físicas del zumo cocristalizado}

Al zumo cocristalizado se le realizaron los siguientes análisis:

Humedad: se determinó por gravimetría con secado en un horno por convección a $110{ }^{\circ} \mathrm{C}$ durante $24 \mathrm{~h}$ (método AOAC 20.013 (AOAC, 1980)). Las mediciones de peso se hicieron en una balanza analítica (con precisión de $0,001 \mathrm{~g})$.

Solubilidad: se determinó midiendo el tiempo requerido para la disolución completa de $10 \mathrm{~g}$ del producto en $100 \mathrm{ml}$ de agua destilada a $25{ }^{\circ} \mathrm{C}$ contenida en una probeta graduada. La disolución se mantuvo en constante agitación constante a $50 \mathrm{rpm}$, usando un agitador mecánico portátil. El tiempo se midió con un cronómetro, hasta que no se observaron sólidos en el líquido (Beristain et al., 1996).

Actividad del agua: la actividad de agua de los polvos se determinó mediante un equipo de actividad de agua, a temperatura de $25^{\circ} \mathrm{C}$, bajo un tiempo de observación de dos minutos.

Densidad aparente: se midió de acuerdo con la metodología reportada por Beristain et al. (1996). Se determinó el volumen ocupado por aproximadamente $30 \mathrm{~g}$ de polvo, depositado en una probeta graduada de $250 \mathrm{ml}$.

Ángulo de reposo: para la medición del ángulo de reposo, el material se introdujo en un embudo metálico con $10 \mathrm{~cm}$ de diámetro, se incorporó en una plataforma metálica y se recogió en una caja de petri. La cantidad de muestra utilizada correspondía a la necesaria para cubrir la placa inferior formando un cono, se utilizó aproximadamente 200 g. Con los valores del radio de placa de Petri y la altura del cono formado por el polvo, se determinó el ángulo de reposo (Bhandari et al., 1998). La misma cantidad de muestra, altura del embudo y placas de petri se utilizaron para todos los experimentos, y las mediciones se realizaron por triplicado. 


\section{Diseño experimental y análisis}

Para estudiar el efecto del pH (3,8 y 4,5), concentración del zumo (50 y $60{ }^{\circ}$ Brix) y porcentaje de zumo concentrado adicionado al jarabe de sacarosa (10 y 20\%), sobre las propiedades físicas de los cocristales de zumo de naranja agria (humedad, solubilidad, actividad de agua, densidad aparente y ángulo de reposo), se empleó un diseño de Box-Behnken, con tres puntos centrales y una réplica. Este es un diseño de segundo orden rotativo basado en un diseño factorial incompleto. Un análisis de varianza (ANOVA) se realizó para determinar los efectos significativos. Se aplicó un modelo polinomial de segundo orden (ecuación 1) para predecir el comportamiento experimental de las variables de repuesta. Las gráficas de superficie de repuesta y el análisis del diseño se realizaron utilizando el programa estadístico JMP 9.0.1 de SAS.

$\widehat{Y}=\beta_{0}+\sum_{i=1}^{k} \beta_{i} X_{i}+\sum_{i=1}^{k} \beta_{i i} X_{i}^{2}+\sum \sum_{i<j=1}^{k} \beta_{i i} X_{i} X_{j}$

donde $\hat{y}$ es el valor predicho de la variable de repuesta; $\beta_{0}, \beta_{\mathrm{i}}, \beta_{\mathrm{ii}}$, y $\beta_{\mathrm{ij}}$ son los coeficientes de regresión para la intercepción, lineal, cuadrática y efectos de interacción, respectivamente; $\mathrm{k}$ es el número de parámetros independientes ( $\mathrm{k}=3$ en este estudio), y $\mathrm{X}_{\mathrm{i}}, \mathrm{X}_{\mathrm{j}}$ son los niveles codificados de las condiciones experimentales.

\section{Resultados y discusión}

La adición de $20 \%$ de zumo de naranja agria con $50^{\circ}$ Brix produjo cocristales con bajos contenido de humedad $(2,59 \%)$, actividad de agua $(0,52)$ y tiempo de solubilidad $(69,4 \mathrm{~s})$. El zumo de naranja agria concentrado con $\mathrm{pH}$ de 4,5 ocasionó cocristales con bajos contenidos de humedad (1,96\%). Los cocristales del zumo de naranja agria mostraron características deseables para un producto en polvo tales como: alta solubilidad y buena fluidez. Sin embargo, presentaron alta humedad $(2,5$ a 4,5\%) y actividad de agua $(0,508$ a 0,798). La cocristalización de zumo de naranja agria ofrece una alternativa para la elaboración de productos de origen natural y con alto valor nutricional; sin embargo, es necesario realizar estudios relacionados con las propiedades funcionales de estos cocristales.

Se pudo apreciar visualmente que todas las muestras obtenidas presentaron diferencias en el color, textura y morfología. En la Figura 1 se presentan varios tratamientos de zumo de naranja agria cocristalizado antes de ser molturados, a diferentes condiciones de pH, sólidos solubles (SS) y zumo adicionado (ZA).

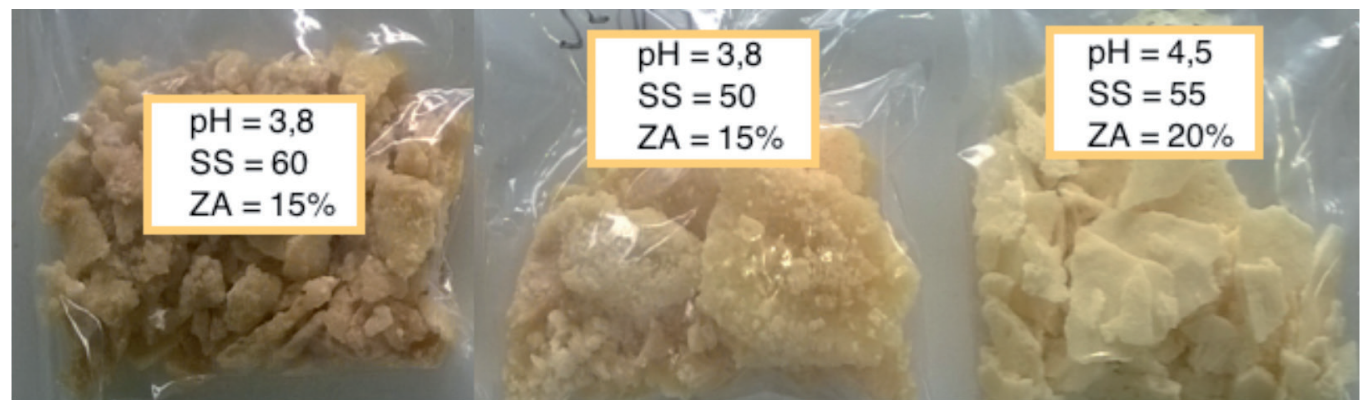

Figura 1. Zumo de naranja agria (Citrus aurantium L.) cocristalizado después del secado. Montería, Colombia. 2015.

SS: sólidos solubles, ZA: zumo adicionado.

Figure 1. Bitter orange (Citrus aurantium L.) juice co-crystallized after drying. Monteria, Colombia. 2015. SS: soluble solids, ZA: juice added. 


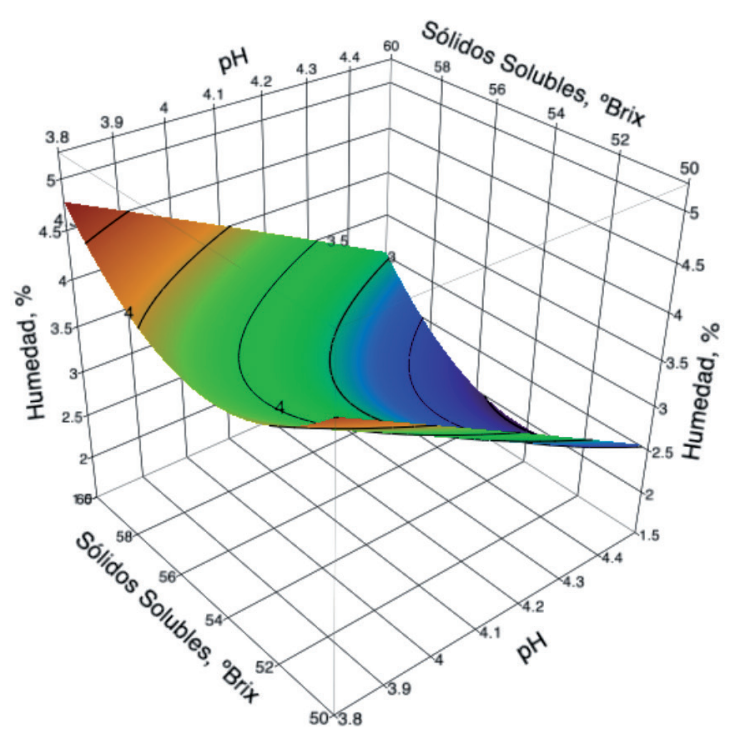

(a)

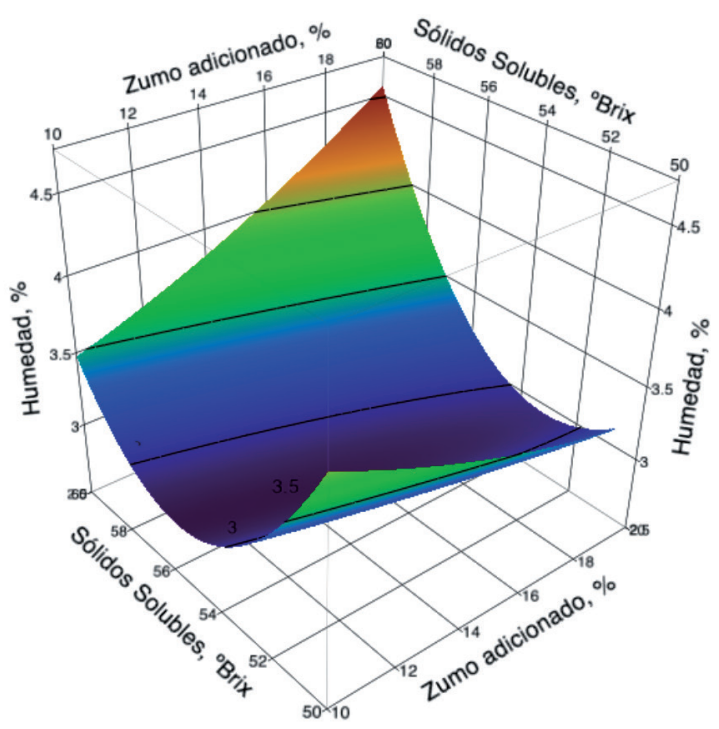

(b)

Figura 2. Superficie de respuesta de humedad en función del (a) pH y los sólidos solubles, (b) del zumo adicionado y los sólidos solubles en zumo de naranja agria (Citrus aurantium L.) cocristalizado. Montería, Colombia, 2015.

Figure 2. Response surface of moisture as a function of (a) $\mathrm{pH}$ and soluble solids, (b) juice added and soluble solids in bitter orange (Citrus aurantium L.) juice co-crystallized. Monteria, Colombia, 2015.

\section{Humedad}

El análisis de varianza (ANOVA) mostró que los factores $\mathrm{pH}(\mathrm{p}=0,001)$, sólidos solubles $(\mathrm{p}=0,0052)$, el factor cuadrático de los sólidos solubles $(\mathrm{p}=0,001)$, y la interacción sólidos solubles - zumo adicionado ( $\mathrm{p}=0,0001$ ), tuvieron un efecto significativo sobre la humedad de los cocristales de zumo de naranja agria, con un nivel de confianza del 95\%. En la Figura 2a se observa un reducción en los valores de humedad a medida que el pH aumentó $(3,7$ a 4,5$)$, la cual es de aproximadamente un 50\%. Esta tendencia concuerda con los datos reportados para jugo de maracuyá (Montes et al., 2006) y extracto de flor de jamaica (Beristain et al., 1994). Esto se pudo deber a que a niveles altos de $\mathrm{pH}$ la adición de hidróxido de calcio es mayor, lo cual provoca un aumento de los sólidos totales que reduce la cantidad de agua disponible para la evaporación durante el secado.

La humedad de los cristales en función de los efectos significativos codificados ( $\mathrm{pH}$ y sólidos solubles) se muestra en la ecuación 2; donde se aprecia que el factor que más influyó sobre la humedad fue el pH (coeficiente mayor). A concentraciones de sólidos solubles de $50{ }^{\circ}$ Brix, un aumento en la cantidad de zumo adicionada (10 a 20\%) causó una disminución de la humedad de aproximadamente un 23\% (Figura 2b), mientras que a concentraciones de sólidos solubles de $60{ }^{\circ}$ Brix, un aumento del zumo adicionado provocó un aumento de la humedad de los cocristales de aproximadamente $25 \%$ (3,48 a 4,39\%). Lo que probablemente esté relacionado con que a bajos contenidos de sólidos solubles se forman conglomerados de mayor porosidad que favorecen la pérdida de humedad de los cocristales durante el proceso de secado (Mendes et al., 2003).

$\%$ Humedad $=2,88-0,92 \mathrm{pH}+0,196 \mathrm{SS}+0,45 \mathrm{SS} * \mathrm{ZA}+0,857 \mathrm{SS}^{2}$ 


\section{Solubilidad}

La solubilidad de los cocristales de zumo de naranja agria estuvo en el rango de 68 a 88 s. Estos valores concuerdan con los reportados para extracto de yerba mate (Ilex paraguariensis), de 79 a $105 \mathrm{~s}$ (Deladino et al., 2007). Sin embargo, son inferiores a los de oleorresina cardamomo cocristalizada ( 110 s) (Sardar y Singhal, 2013) y superiores a los de otros productos cocristalizados, tales como: jugo de maracuyá, 40 a $60 \mathrm{~s}$ (Astolfi et al., 2005; Montes et al., 2006), sacarosa, 45 s (López-Córdoba et al., 2014), y extracto de flor de Jamaica (Hibiscus sabdarifa L.) de 28,3 - 35 s (Beristain et al., 1994). Esta diferencia se pudo deber al tamaño de los cristales y a la alta concentración del zumo de naranja agria utilizado (50 a $60{ }^{\circ}$ Brix).

El ANOVA reveló diferencia significativa, a nivel de confianza del 95\%, del efecto cuadrático del zumo adicionado $(\mathrm{p}=0,0169)$, y de las interacciones $\mathrm{pH}$-zumo adicionado $(\mathrm{p}=0,0489)$ y sólidos solubles-zumo adicionado $(\mathrm{p}=0,0079)$ sobre la solubilidad. A concentraciones de sólidos solubles de $50{ }^{\circ}$ Brix, la solubilidad se redujo aproximadamente un 17\%, cuando el zumo adicionado pasó de 10-20\% (Figura 3a), mientras que a concentraciones de $60{ }^{\circ}$ Brix se presentó un aumento de esta propiedad en aproximadamente un 20\% (68,9 a 82,69s). Estas tendencias probablemente estén relacionadas con la cantidad de sólidos solubles adicionados (50 a $60{ }^{\circ} \mathrm{Brix}$ ). Los valores bajos de solubilidad de los cocristales se pueden atribuir al hecho de que estos son sistemas de aglomerados porosos, en los cuales las soluciones acuosas pueden penetrar fácilmente y liberar el compuesto activo para su disolución (Chen et al., 1988).

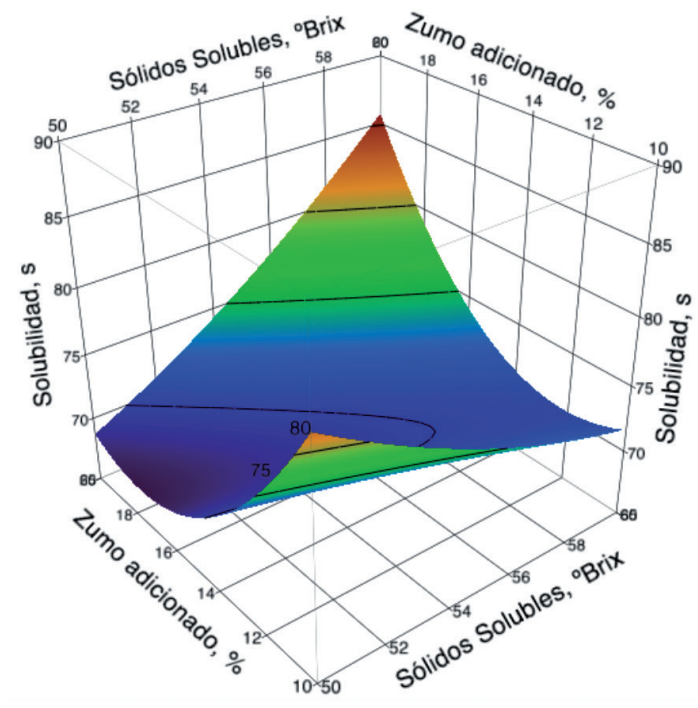

(a)

Figura 3. Superficie de respuesta de la solubilidad en función adicionado y pH en zumo de naranja agria (Citrus aurantium L.) cocristalizado. Montería, Colombia. 2015.

Figure 3. Response surface of solubility as a function of (a) juice added and soluble solids, (b) juice added and $\mathrm{pH}$ in bitter orange (Citrus aurantium L.) juice co-crystallized. Monteria, Colombia. 2015.

La solubilidad de los cristales de zumo de naranja agria con respecto a los factores significativos codificados (pH y ZA: zumo adicionado) se presenta en la ecuación 3. La interacción del factor pH-zumo adicionado, se puede 
apreciar en la Figura 3b. A pH de 3,8 un aumento de la cantidad de zumo adicionado causó un aumento del 13,7\% de la solubilidad (70,9 a 80,7s), mientras que a pH de 4,5 esta propiedad disminuyó un 12,1\% (80,7 a 70,9s), cuando se produjo un aumento en el zumo adicionado (10 a 20\%).

Solubilidad $=69,5-4,88 \mathrm{pH} * \mathrm{ZA}+6,88 \mathrm{SS} * \mathrm{ZA}+6,31 \mathrm{ZA}^{2}$

\section{Actividad del agua}

Los valores de actividad de agua $\left(\mathrm{a}_{\mathrm{w}}\right)$ estuvieron entre 0,508 y 0,798 , similares a los reportados para oleorresina cardamomo cocristalizada $(\sim 0,52)$ (Sardar y Singhal, 2013); pero superiores a los reportados para sacarosa $(0,473$ ) y extracto de yerba mate cocristalizado $(0,460)$ (Deladino et al., 2007), lo cual se debió a los altos contenidos de humedad ( 2 a $5 \%$ ) que presentaron los cocristales de zumo de naranja agria. Estos valores de $\mathrm{a}_{\mathrm{w}}$ fueron superiores a 0,47, lo que puede ocasionar problemas de apelmazamiento y de seguridad microbiológica (Deladino et al., 2007).

En el ANOVA hubo diferencia significativa $(\mathrm{p}<0,05)$ para el factor zumo adicionado $(\mathrm{p}=0,0015)$, el factor cuadrático sólidos solubles $(\mathrm{p}=0,0009)$ y la interacción $\mathrm{pH}$-zumo adicionado $(\mathrm{p}=0,0285)$. En la ecuación 4 se presenta la actividad de agua $\left(\mathrm{a}_{\mathrm{w}}\right)$ en función de los factores significativos codificados ( $\mathrm{pH}$, SS: sólidos solubles, ZA: zumo adicionado).

$a_{w}=0,67-0,05 \mathrm{ZA}-0,046 \mathrm{pH} * \mathrm{ZA}-0,078 S S^{2}$

En las Figuras 4a y 4b, se observa una tendencia a disminuir la actividad de agua $(\sim 14 \%)$, a medida que la proporción de zumo adicionado aumentó. Al someter el jarabe de sacarosa a altas temperaturas y tiempos prolongados, la sacarosa tendió a invertirse en dextrosa y fructosa, las cuales promueven una mayor

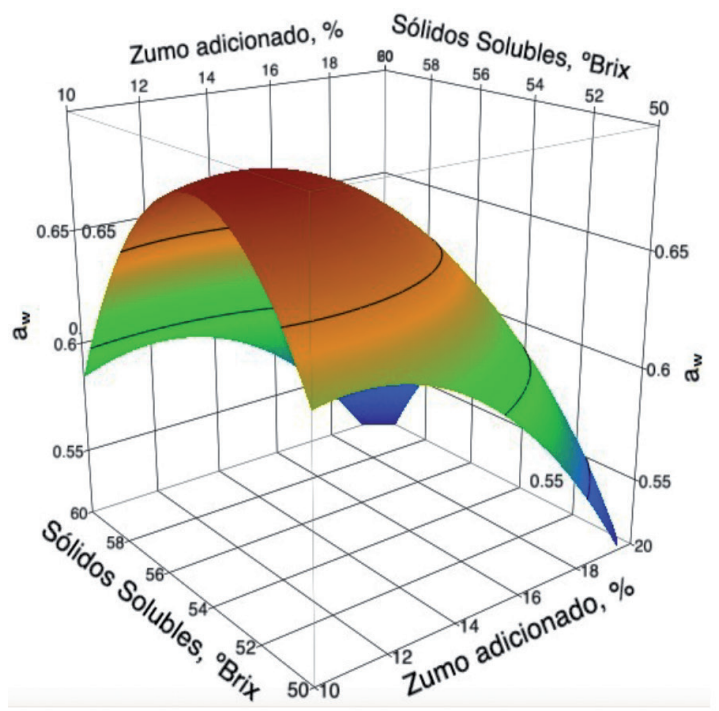

(a)

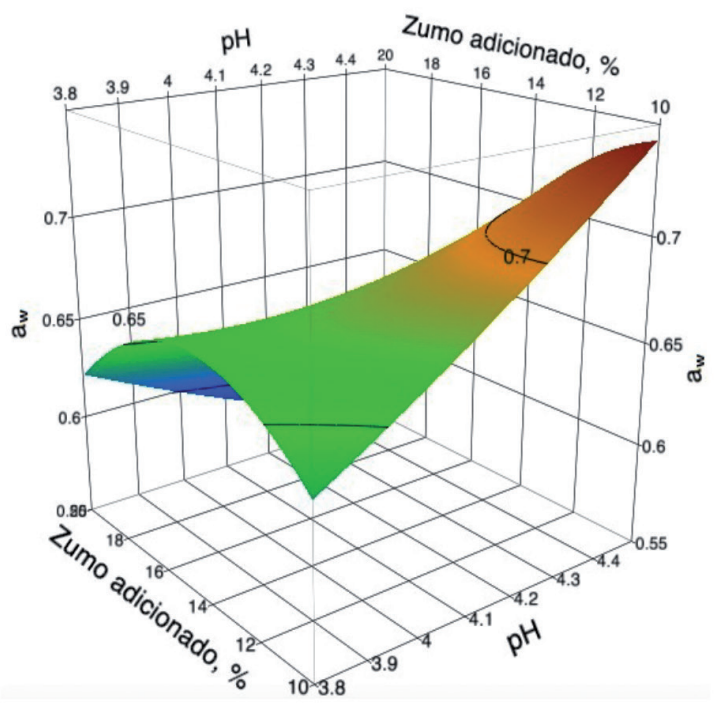

(b)

Figura 4. Superficie de respuesta de la actividad de agua en función del (a) zumo adicionado y sólidos solubles, (b) zumo adicionado y pH en zumo de naranja agria (Citrus aurantium L.) cocristalizado. Montería, Colombia, 2015.

Figure 4. Response surface of water activity as a function of (a) juice added and soluble solids, (b) juice added and $\mathrm{pH}$ in bitter orange (Citrus aurantium L.) juice co-crystallized. Monteria, Colombia, 2015. 
higroscopicidad en los cocristales, debido a su posible encapsulación. Esta velocidad de inversión probablemente se vio reducida debido a que al momento de mezclar el jarabe de sacarosa con el zumo concentrado, se sobresaturó el sistema por las altas concentraciones de azúcares (Dávila, 2004).

En la Figura 4b se puede apreciar la interacción que existió entre los factores zumo adicionado y pH. Cuando se adicionó un $10 \%$ de zumo un aumento en el pH ocasionó un aumento de la $\mathrm{a}_{\mathrm{w}}$ de un 13,5\% (0,67 a 0,77), mientras que cuando se adicionó un 20\% de zumo de naranja, el aumento del pH causó una disminución del 13,7\% (0,67 a 0,57) en la $a_{w}$. Cabe destacar que, en ciertas condiciones, la sacarosa puede sufrir una hidrólisis (inversión), produciendo una molécula de glucosa y una de fructosa. La inversión es controlada por el $\mathrm{pH}$ (favorecida en soluciones levemente ácidas). La fructosa presenta mayor higroscopicidad y, por tanto, acarrea absorción de mayor cantidad de agua, que disolverá más sacarosa y que será hidrolizada subsecuentemente.

\section{Densidad aparente}

Los valores de densidad aparente (D.a) de los cocristales de zumo de naranja agria estuvieron comprendidos entre 500 y $653 \mathrm{~kg} / \mathrm{m}^{3}$, densidades similares se han reportado para diversos productos cocristalizados: extracto de yerba mate $\left(697 \mathrm{~kg} / \mathrm{m}^{3}\right)$ (Deladino et al., 2007) y el aceite de cáscara de naranja $\left(674,4 \mathrm{~kg} / \mathrm{m}^{3}\right)$ (Beristain et al., 1996). La mayoría de los polvos alimenticios están en el rango de 300 a $800 \mathrm{~kg} / \mathrm{m}^{3}$; por ejemplo, el café instantáneo $(330 \mathrm{~kg} /$ $\left.\mathrm{m}^{3}\right)$, las harinas $\left(449 \mathrm{~kg} / \mathrm{m}^{3}\right)$, el almidón de maíz (Zea mays) $\left(560 \mathrm{~kg} / \mathrm{m}^{3}\right)$, la leche $\left(610 \mathrm{~kg} / \mathrm{m}^{3}\right)$ y el azúcar granulada (sacarosa) $\left(800 \mathrm{~kg} / \mathrm{m}^{3}\right)$ (Lewis, 2006).

El ANOVA reveló que existió diferencia significativa $(\mathrm{p}<0,05)$ para los factores $\mathrm{pH}(\mathrm{p}=0,0188)$, zumo adicionado ( $\mathrm{p}=0,0119)$, el factor cuadrático $\mathrm{pH}(\mathrm{p}=0,0047)$, y las interacciones $\mathrm{pH}$-sólidos solubles del zumo $(\mathrm{p}=0,0004)$ y zumo adicionado-sólidos solubles $(\mathrm{p}=0.0001)$. La densidad aparente en función de los factores significativos codificados se presenta en la ecuación 5.

D.a $=615,1-12,9 p H-13,2 \mathrm{ZA}+30,0 \mathrm{pH} * \mathrm{SS}+34,5 \mathrm{SS} * \mathrm{ZA}+23,1 p H^{2}$

A niveles bajos de sólidos solubles del zumo (50 ${ }^{\circ}$ Brix) y porcentaje de zumo adicionado (10\%), se presentaron los valores más altos de densidad aparente $\left(680 \mathrm{~kg} / \mathrm{m}^{3}\right)$ (Figura $\left.5 \mathrm{a}\right)$. Esto probablemente se debió a que al haber menos sólidos a encapsular, la sacarosa cristalizó mejor, fue más compacta y formó caras más planas, y así se obtuvo un producto menos poroso (Astolfi et al., 2005).

A cantidades bajas de zumo adicionado (10\%), un aumento de los sólidos solubles produjo una disminución de la densidad del 10,4\%; mientras que cuando el zumo de naranja agria adicionado fue del 20\%, un aumento en los sólidos solubles causó un aumento del 12,2\% en la densidad de los cocristales de zumo de naranja agria.

\section{Ángulo de reposo}

Los cocristales de zumo de naranja agria presentaron valores de ángulo de reposo $\left(\phi_{\text {rep }}\right)$ comprendidos entre los 34 y $45^{\circ}$, lo cual indica que presentaron propiedades de flujo libre sin problemas de cohesión (Bhandari et al., 1998). Según Chen et al. (1988) el azúcar refinado comercial presenta un ángulo de reposo de 31 a $38^{\circ}$, y al comparar estos valores con los de los cocristales de naranja agria obtenidos, hay que indicar que fueron similares, señalando que este parámetro se encuentra altamente influenciado por las características de flujo del material encapsulante (sacarosa). Por otra parte, estos valores fueron similares a los reportados para varios productos cocristalizados, tales como: miel de abeja $\left(39^{\circ}\right)$ (Bhandari et al., 1998), extracto de yerba mate (40,4²) (López-Córdoba et al., 2014), lactato de calcio $\left(40,4^{\circ}\right)$ (Deladino et al., 2007) y jugo de maracuyá (30-32 $)$ (Astolfi et al., 2005; Montes et al., 2006). 


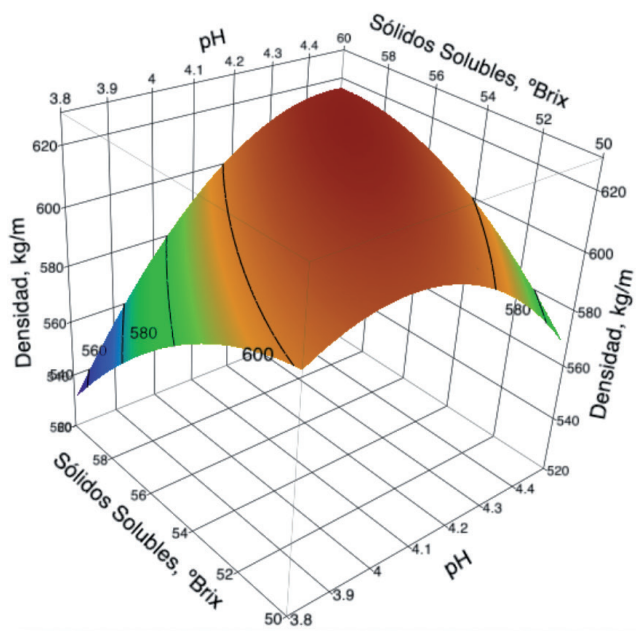

(a)

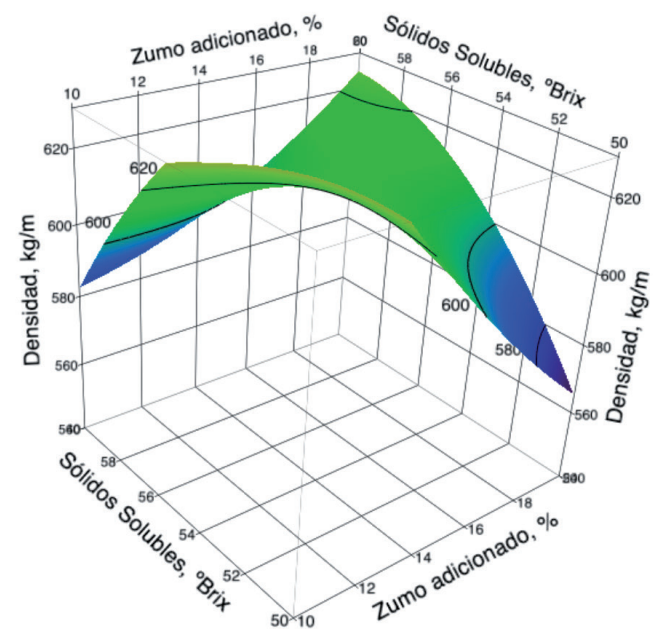

(b)

Figura 5. Superficie de respuesta de densidad aparente en función del (a) pH y sólidos solubles, (b) zumo adicionado y sólidos solubles en zumo de naranja agria (Citrus aurantium L.) cocristalizado. Montería, Colombia, 2015.

Figure 5. Response surface of apparent density as a function of (a) $\mathrm{pH}$ and soluble solids, (b) juice added and soluble solids in bitter orange (Citrus aurantium L.) juice co-crystallized. Monteria, Colombia, 2015.

El ángulo de reposo mostró diferencia significativa $(\mathrm{p}<0,05)$ para los efectos cuadráticos sólidos solubles $(\mathrm{p}=0,0304)$, zumo adicionado $(\mathrm{p}=0,0096)$ y la interacción sólidos solubles - zumo adicionado ( $\mathrm{p}=0,0305)$. En la ecuación 6 se muestra el ánguno de reposo en función de los factores significativos codificados.

$\phi_{\text {rep }}=37,5+01,85 S S * Z A-1,86 S S^{2}+2,31 Z^{2}$

En la Figura 6a se observa el efecto de interacción, a concentraciones bajas de sólidos solubles un aumento en la adición del zumo de naranja agria ocasionó un aumento del 9,3\% del ángulo de reposo, mientras que a sólidos solubles altos este aumento en el zumo de naranja agria adicionado causó una disminución del 10,23\% de esta propiedad. Este comportamiento del ángulo de reposo probablemente se encuentre relacionado con la humedad de los cocristales, la cual mostró la misma tendencia para la interacción zumo adicionado - sólidos solubles. Cabe destacar, que a mayor humedad probablemente exista un ensanchamiento en el área proyectada de los cocristales, lo cual intensifica la fricción interna y a su vez el ángulo de reposo (Dursun y Dursun, 2005).

En la Figura 6b se muestra el efecto cuadrático del zumo adicionado, en el cual se presentó un mínimo del ángulo de reposo cuando la cantidad del zumo adicionado estuvo en aproximadamente un $15 \%$.

\section{Agradecimientos}

Los autores agradecen a la División de Investigaciones de la Universidad de Córdoba, y en especial a la convocatoria "Proyectos de investigación en menor cuantía para financiar trabajos de grado en la modalidad de proyectos de investigación de pregrado" 2015, por el apoyo económico a esta investigación. 

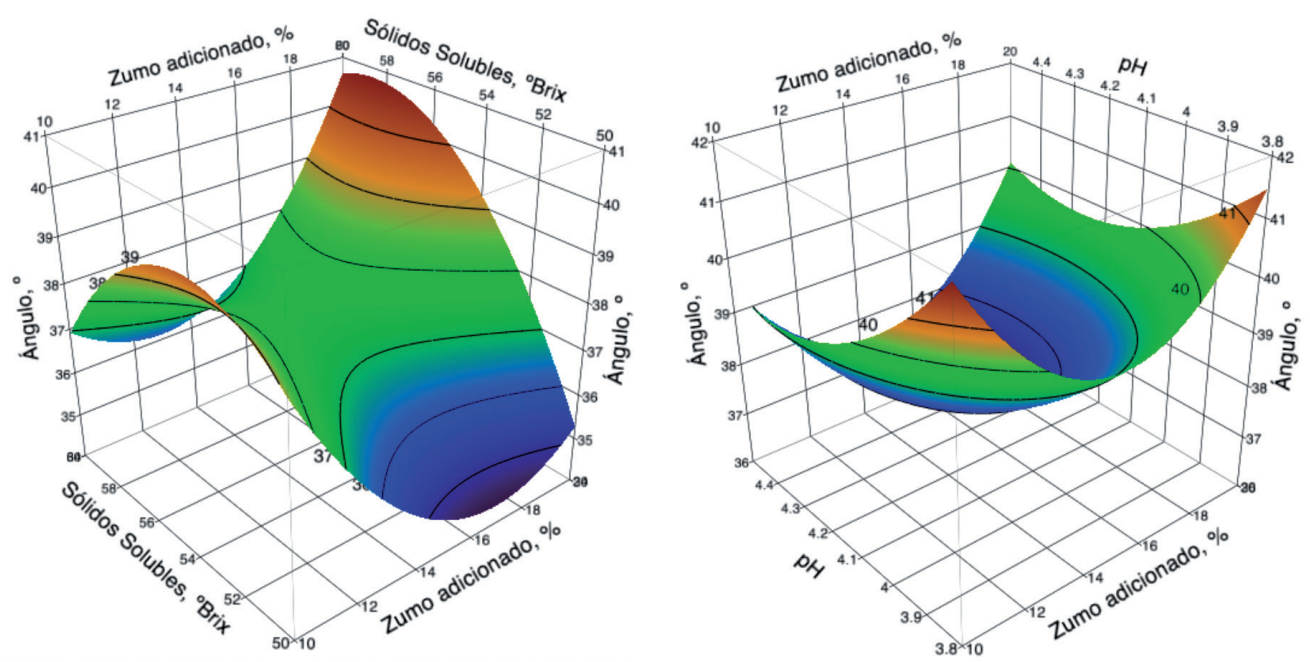

Figura 6. Superficie de respuesta del ángulo de reposo en función del (a) zumo adicionado y sólidos solubles, (b) zumo adicionado y pH en zumo de naranja agria (Citrus aurantium L.) cocristalizado. Montería, Colombia, 2015.

Figure 6. Response surface of angle of repose as a function of (a) juice added and soluble solids, (b) juice added and $\mathrm{pH}$ in bitter orange (Citrus aurantium L.) juice co-crystallized. Monteria, Colombia, 2015.

\section{Literatura citada}

Alonso, J. 2004. Tratado de fitofármacos y nutraceúticos. Editorial Corpus, Rosario, ARG.

AOAC (Association of Official Analytical Chemist). 1980. AOAC: Oficial methods of analysis. AOAC, WA, USA.

Astolfi, Z., A. Souza, É. Reipert, e V. Telis. 2005. Encapsulação de suco de maracujá por co-cristalização com sacarose: cinética de cristalização e propriedades físicas. Ciênc. Tecnol. Aliment. 25:795-801.

Astray, G., J. Mejuto, R. Rial, C. González, and J. Simal. 2009. A review on the use of cyclodextrins in foods. Food Hydrocoll. 23:1631-1640.

Awad, A., and A.C. Chen. 1993. A new generation of sucrose products made by cocrystallization. Food Technol. 47:146-148.

Barón, R., y A. Villa. 2013. Evolución de los parámetros de calidad de naranja valencia producida en el municipio de Chimichagua, Cesar - Colombia. Rev. Temas Agrarios 18:66-74.

Beristain, C., R. Mendoza, and A. Vázquez. 1994. Cocrystallization of Jamaica (Hibiscus sabdarifa L.) granules. Food Sci. Technol. 27:347-349.

Beristain, C., A. Vázquez, H. García, and E. Vernon-Carter. 1996. Encapsulation of orange peel oil by co-crystallization. Food Sci. Technol. 29:645-647.

Bhandari, B., N. Datta, B. D'arcy, and G. Rintoul. 1998. Co-crystallization of honey with sucrose. Food Sci. Technol. 31:138142.

Bhandari, B.R., and R.W. Hartel. 2002. Co-crystallization of sucrose at high concentration in the presence of glucose and fructose. J. Food Sci. 67:1797-1802. 
Chen, A., M. Veiga, and A. Rizzuto. 1988. Co-crystallization and encapsulation process. Food Technol. 44:87-90.

Dávila, J. 2004. Estudio experimental del efecto de la porosidad de partícula sobre el proceso de secado en un lecho fluidizado a vacío empleando aire. Tesis Lic., Universidad de las Américas, Puebla, MEX.

Deladino, L., P. Anbinder, A. Navarro, and M. Martino. 2007. Co-crystallization of yerba mate extract (Ilex paraguariensis) and mineral salts within a sucrose matrix. J. Food Eng. 80:573-580.

Dursun, I., and E. Dursun. 2005. Some physical properties of caper seed. Biosys. Eng. 86:172-177.

Lewis, M.J. 2006. Physical properties of foods and food processing systems. Woodhead Publishing Limited, Cambridge, GBR.

López-Córdoba, A., L. Deladino, L. Agudelo-Mesa, and M. Martino. 2014. Yerba mate antioxidant powders obtained by cocrystallization: Stability during storage. J. Food Eng. 124:158-165.

López-Córdoba, A., S. Matera, L. Deladino, A. Hoya, A. Navarro, and M. Martino. 2015. Compressed tablets based on mineralfunctionalized starch and co-crystallized sucrose with natural antioxidants. J. Food Eng. 146:234-242.

Mendes, M., F.D. Magalhäes, and L.M. Madeira. 2003. Sucrose inversion: An experiment on heterogeneous catalysis. Int. J. Eng. Educ. 19:893-901.

Montes, E., C. De Paula, y F. Ortega. 2006. Encapsulamiento por co-cristalización de jugo de maracuyá (Passiflora edulis). Rev. Temas Agrarios 12:5-12.

Sardar, B.R., K.M. Tarade, and R.S. Singhal. 2013. Stability of active components of Cardamom oleoresin in co-crystallized sugar cube during storage. J. Food Eng. 117:530-537.

Sardar, R., and R.S. Singhal. 2013. Characterization of co-crystallized sucrose entrapped with Cardamom oleoresin Bikash. J. Food Eng. 117:521-529. 